\title{
The Keck Cosmic Web Imager: a capable new integral field spectrograph for the W. M. Keck Observatory
}

Patrick Morrissey

Mateusz Matuszewski

Chris Martin

Anna Moore

Sean Adkins

Harland Epps

Randy Bartos

Jerry Cabak

Dave Cowley

Jack Davis

Alex Delacroix

Jason Fucik

David Hilliard

Ean James

Steve Kaye

Nicole Lingner

James D. Neill

Christoph Pistor 


\title{
The Keck Cosmic Web Imager: a capable new integral field spectrograph for the W. M. Keck Observatory
}

\author{
Patrick Morrissey*a ${ }^{*}$, Mateusz Matuszewski ${ }^{\mathrm{a}}$, Chris Martin $^{\mathrm{a}}$, Anna Moore $^{\mathrm{b}}$, Sean Adkins ${ }^{\mathrm{c}}$, \\ Harland Epps ${ }^{\mathrm{d}}$, Randy Bartos ${ }^{\mathrm{e}}$, Jerry Cabak ${ }^{\mathrm{d}}$, Dave Cowley ${ }^{\mathrm{d}}$, Jack Davis ${ }^{\mathrm{b}}$, Alex Delacroix ${ }^{\mathrm{b}}$, \\ Jason Fucik ${ }^{\mathrm{b}}$, David Hilliard ${ }^{\mathrm{d}}$, Ean James ${ }^{\mathrm{c}}$, Steve Kaye ${ }^{\mathrm{b}}$, Nicole Lingner ${ }^{\mathrm{a}}$, James D. Neill ${ }^{\mathrm{a}}$, \\ Christoph Pistor ${ }^{\mathrm{d}}$, Drew Phillips ${ }^{\mathrm{d}}$, Connie Rockosi ${ }^{\mathrm{d}}$, and Bob Weber ${ }^{\mathrm{b}}$ \\ ${ }^{a}$ MS278-17, Caltech, 1200 E. California Blvd., Pasadena, CA, USA 91125 \\ ${ }^{b}$ MS11-17, Caltech Optical Observatories, 1200 E. California Blvd., Pasadena, CA, USA 91125 \\ ${ }^{c}$ W. M Keck Observatory, 65-1120 Mamalahoa Highway, Kamuela, HI, USA 96743 \\ ${ }^{\mathrm{d} U C O / L i c k ~ O b s e r v a t o r y, ~} 1156$ High St., Santa Cruz, CA, USA 95062 \\ ${ }^{\mathrm{e}}$ Jet Propulsion Laboratory, California Institute of Technology, Pasadena, CA, USA 91109
}

\begin{abstract}
The Keck Cosmic Web Imager (KCWI) is a new facility instrument being developed for the W. M. Keck Observatory and funded for construction by the Telescope System Instrumentation Program (TSIP) of the National Science Foundation (NSF). KCWI is a bench-mounted spectrograph for the Keck II right Nasmyth focal station, providing integral field spectroscopy over a seeing-limited field up to 20"x33" in extent. Selectable Volume Phase Holographic (VPH) gratings provide high efficiency and spectral resolution in the range of 1000 to 20000. The dual-beam design of KCWI passed a Preliminary Design Review in summer 2011. The detailed design of the KCWI blue channel (350 to $700 \mathrm{~nm})$ is now nearly complete, with the red channel $(530$ to $1050 \mathrm{~nm})$ planned for a phased implementation contingent upon additional funding. KCWI builds on the experience of the Caltech team in implementing the Cosmic Web Imager (CWI), in operation since 2009 at Palomar Observatory. KCWI adds considerable flexibility to the CWI design, and will take full advantage of the excellent seeing and dark sky above Mauna Kea with a selectable nod-and-shuffle observing mode. In this paper, models of the expected KCWI sensitivity and background subtraction capability are presented, along with a detailed description of the instrument design. The KCWI team is lead by Caltech (project management, design and implementation) in partnership with the University of California at Santa Cruz (camera optical and mechanical design) and the W. M. Keck Observatory (program oversight and observatory interfaces).
\end{abstract}

Keywords: Integral Field Spectrograph Keck Observatory KCWI

\section{INTRODUCTION}

The Keck Cosmic Web Imager (KCWI) is a new integral field spectrograph (Martin, 2010) that is being developed for the W. M. Keck Observatory on Mauna Kea, HI. This spectrograph is an adaptation of the Palomar Cosmic Web Imager (CWI), which has been operating since 2009. CWI (Matuszewski, 2010) was funded by the National Science Foundation (NSF) for the primary objective of measuring low surface brightness blue emission from a moderately-sized (40" x 60") field at a spectral resolution of 5000. By contrast, KCWI is intended as a facility instrument for the Keck II telescope and will add features to the CWI design that allow a wide range of science topics to be efficiently addressed. A sample of these includes measurement of emission from the intergalactic medium (cosmic web), cosmic evolution from $\mathrm{z} \sim 2$ to 3 , black holes at the center of globular clusters, and high-z emission from the era of re-ionization.

KCWI will be bench-mounted at the right Nasmyth focus of the Keck II telescope. An image de-rotator and tracking guider maintain the orientation of science targets at the focal plane. The instrument employs a dual beam design and detectors optimized separately for the red and the blue to attain high efficiency across the visible spectrum (350 to 1050

*patrick@srl.caltech.edu

Ground-based and Airborne Instrumentation for Astronomy IV, edited by lan S. McLean, Suzanne K. Ramsay, Hideki Takami, Proc. of SPIE Vol. 8446, 844613 · @ 2012 SPIE · CCC code: 0277-786X/12/\$18 · doi: 10.1117/12.924729 
$\mathrm{nm}$ ). One of the chief innovations of the instrument is a configurable Integral Field Unit (IFU) that contains three selectable image slicers providing a range of slit widths. Coupled with a suite of exchangeable gratings, KCWI will achieve spectral resolution from 1000 to 20000 over a field of view from $20^{\prime \prime} \times 33$ " to 20 " x 8", respectively. KCWI also incorporates a selectable nod-and-shuffle $(\mathrm{N} \& \mathrm{~S})$ mask, which covers $2 / 3$ of the CCD active area when deployed to enable two patches of sky to be observed alternately (one patch of sky is hidden under the mask while the other is observed) during the course of a single integration for improved background subtraction with only a single detector readout.

The purpose of this paper is to review the science motivation for KCWI and the flow-down to requirements, and to present models of the expected KCWI sensitivity and background subtraction capability given several possible forms of signal variability. We show how the planned nod and shuffle observing strategy can improve systematic sky subtraction to the desired level, enabling measurements of emission fainter than $1 \%$ of the sky background. We review progress on the design of the blue channel during the on-going detailed design phase.

KCWI is funded for construction by the NSF Telescope System Instrumentation Program (TSIP). This program has supported KCWI through a successful preliminary design review in 2011 of the integrated instrument with both red and blue channels. Current funding supports detailed design and construction of the blue channel only. The red channel will be completed in a phased implementation with future funds, which are actively being sought. The KCWI team is lead by Caltech (project management, optics, mechanical design, systems engineering) in collaboration with UCO/Lick Observatory (camera optical and mechanical design) and the W. M. Keck Observatory (program oversight and observatory interfaces). The IFU and image slicer exchange mechanism will be fabricated by Winlight Optics (France).

\section{KCWI SCIENCE MOTIVATION AND REQUIREMENTS}

KCWI is intended to provide flexibility for a wide range of science applications ranging from observations of faint diffuse emission at a fraction of sky brightness to galaxy kinematics and dynamics in globular clusters. This wide range of observational parameters is addressed by a high efficiency, flexible and configurable instrument design that leverages our experience with CWI at Palomar. Our top-level requirements, shown in Table 1, result from the desire to satisfy community observing needs, while our phased implementation plan beginning with blue channel implementation is cognizant of a realistic funding profile.

The KCWI requirements can be viewed as stemming from a baseline design intended to measure emission from the intergalactic medium (cosmic web), with enhancements intended to satisfy a larger community of astronomers. For reference, a theoretical model of cosmic web emission is presented in Figure 1, excerpted from Bertone and Schaye (2012). The brightest features are of order $1000 \mathrm{LU}\left(1 \mathrm{LU}=1\right.$ photon- $\left.\mathrm{cm}^{-2}-\mathrm{s}^{-1}-\mathrm{sr}^{-1}\right)$. It is instructive to review the requirements that follow from the flux estimates presented here. The angular scale of the features is reasonably matched by the KCWI field of view, while the surface brightness places some demanding requirements on systematic background subtraction. Consider the sensitivity estimates presented in Table 2, which are representative of our expectations for KCWI at the dark Mauna Kea summit. We show the contributions of sky background (B band sky brightness of 23 magnitudes per square arcsecond), detector dark current and read noise in stacks of 30 minute KCWI images for scales from 0.3 " to 30 " over a range of total integration time from 1 to 30 hours across a single spectral line at a resolution of 5000. The detection limits possible with sufficiently accurate sky subtraction will range from better than $100 \mathrm{LU}$ for the full slicer in 30 hours to about 40000 LU per $2 \times 2$ pixel in a one hour exposure. Shot noise from the continuum sky background is the dominant source of noise in all of these circumstances and ultimately sets the detection limit of the instrument, defined here as 5 times the total noise in a stacked image. The key points to appreciate are as follows: 1) the anticipated detection limit of KCWI in "light bucket" mode (integrating the combined light of the entire slicer into a single spectrum) is 100 times fainter than the brightest modeled cosmic web features and 2) achieving the KCWI "light bucket" detection limit requires excellent sky subtraction, of order $1 \times 10^{-4}$ of the sky brightness (about 10x lower than the detection limit).

Measurement of the sky at this level requires consideration of the elements of time variation that might occur during combined integrations of up to 30 hours. The natural sky variation is the most obvious source, but there can be significant instrumental variations as well. Two examples result from the required rotation of the reflective K-mirror image de-rotator at the entrance of the instrument. The first is the variation in measured intensity of the polarized sky as 
the K-mirror rotates, and the second is variation in vignetting of the hexagonal telescope pupil at the K-mirror rotates. We will review these in some detail and show how our proposed mode of observing is expected to eliminate the resulting systematics.

Table 1: KCWI Top Level Science Requirements

\begin{tabular}{|c|c|c|}
\hline Science Requirement & Value & Instrument Requirement \\
\hline Spatial Resolution & $\begin{array}{l}\text { Seeing-limited x Slit-width limited } \\
\qquad 0.5^{\prime \prime} \times(0.35 \text { to } 1.4)^{\prime \prime}\end{array}$ & $\begin{array}{c}\text { Multiple image slicers } \\
\text { Aberration-corrected optics } \\
\text { Instrument stability }\end{array}$ \\
\hline Field of View & $20 " \times(8$ to 33$) "$ & Integral Field Spectroscopy \\
\hline Spectral Resolution & 1000 to 20000 & $\begin{array}{l}\text { Selectable gratings and slicers } \\
\text { Aberration-corrected optics } \\
\text { Instrument Stability }\end{array}$ \\
\hline Bandpass & 0.35 to 1.05 microns & Dual channel design \\
\hline Efficiency & $>0.25$ & $\begin{array}{l}\text { Dual channel design } \\
\text { Multilayer Ag coatings }\end{array}$ \\
\hline Sky/object subtraction & $0.01 \%$ sky & Nod-and-shuffle Mode \\
\hline Object contrast & $>1000: 1$ at $r>5 "$ & $\begin{array}{l}\text { Baffling } \\
\text { Low surface roughness }\end{array}$ \\
\hline Flexibility & Accommodates a broad range of science & $\begin{array}{c}\text { Selectable gratings } \\
\text { Selectable image slicers }\end{array}$ \\
\hline Operations & Simple & $\begin{array}{l}\text { Real time data reduction } \\
\text { Scriptable nod-and-shuffle }\end{array}$ \\
\hline
\end{tabular}

KCWI will employ a "nod and shuffle" (N\&S) mode of observing to reduce systematics similar to the mode described by Glazebrook and Bland-Hawthorn (2001). In the KCWI implementation, a deployable mask will cover $2 / 3$ of the CCD, allowing the central $1 / 3$ to be illuminated by the spectrograph. The mask reduces $2 / 3$ of the spectral bandwidth, but retains the full field of view of the instrument. During KCWI cosmic web observations, the telescope will be alternately nodded back and forth between a "science" field and one or more background fields. A graphical depiction of this mode is shown in Figure 2. The N\&S technique we plan to implement involves collecting both science and background field measurements during a single exposure. The sequence begins and ends with half-length background exposures, while the middle of the exposure is comprised of science and background pairs of equal length. A typical sequence might involve a 1-minute background field integration followed by 19 2-minute integrations, each separated by a "nod" of the telescope from one field to the other and a "shuffle" of charge on the CCD. The sequence completes with a final 1-minute background field exposure. Including overhead, the example sequence takes approximately 45 minutes. The advantage is that only one readout noise penalty is incurred at the end of the sequence, which naturally balances linear time variations in the sky background across the two fields.

We have investigated the ability of our planned N\&S sequence to eliminate natural sky variations. In Figure 3, we show the model N\&S sky subtraction precision for a range of observing cadences from 1 to 10 minutes per field (with the same combined 40 minute observing time) in the presence of a sinusoid sky variation of $+/-10 \%$ amplitude across a range of variation periods. The sequence results in subtraction residuals at the desired level $\left(\sim 1 \times 10^{-4}\right)$ as long as the sky variations are smooth and slowly varying throughout the night. High frequency variations induce larger residuals because the observing cadence is not able to balance the variation in the two fields. A slow B-band sky variation of the 
form we used is consistent with the data presented in Patat (2008). Extremely faint levels could be reached by "cherrypicking" data from the quietest nights.

Table 2: A detailed accounting of the major noise sources that define the KCWI sensitivity. Assumption include a system efficiency of $0.25,2 \times 2$ pixel binning $(1$ physical pixel $=0.15 ")$, read noise $=3$ electrons, frame time $=30$ minutes, spectral resolution $=5000$, central wavelength $=0.44$ microns, and $B$ band sky brightness $=23$ magnitudes per square arcsecond.

\begin{tabular}{l|cc|cc}
\hline \multicolumn{1}{c|}{ Component } & \multicolumn{2}{|c|}{$\mathbf{2 \times 2}$ pixel } & \multicolumn{2}{c}{ Full Slicer } \\
\hline Exposure time (hours) & 1 & 30 & 1 & 30 \\
Sky signal (electrons) & 48.93 & $1.47 \times 10^{3}$ & $3.71 \times 10^{5}$ & $1.11 \times 10^{7}$ \\
Sky shot noise (electrons) & 7 & 38.31 & 608.95 & $3.34 \times 10^{3}$ \\
\hline CCD dark (electrons) & 4 & 120 & $3.03 \times 10^{4}$ & $9.09 \times 10^{5}$ \\
CCD dark noise (electrons) & 2 & 10.95 & 174.11 & 953.64 \\
Read noise (electrons) & 4.24 & 23.24 & 369.34 & $2.02 \times 10^{3}$ \\
Total noise (electrons) & 8.42 & 46.13 & 733.17 & $4.02 \times 10^{3}$ \\
\hline Detection limit (5*noise, electrons) & 42.11 & 230.65 & $3.67 \times 10^{3}$ & $2.01 \times 10^{4}$ \\
\hline Detection limit/Sky signal & 0.86 & 0.16 & $9.89 \times 10^{-3}$ & $1.8 \times 10^{-3}$ \\
\hline Detection limit (LU) & $\mathbf{3 . 3 \times 1 0 ^ { 4 }}$ & $\mathbf{6 \times 1 0 ^ { 3 }}$ & $\mathbf{3 8 3}$ & $\mathbf{7 0}$ \\
\hline
\end{tabular}

Instrumental effects have the potential to be as significant as the natural sky variation. For example, the KCWI K-mirror image de-rotator induces polarization into the input beam, and can present a variable background intensity if the sky is polarized. In order to investigate this effect, we have modeled the rotation of the K-mirror during KCWI observations through a range of object declination angles. The K-mirror will rotate at $1 / 2$ the rate of the parallactic angle for a given object at the time it is observed, because a rotation of the K-mirror induces image rotation at twice its angular rate. Following Filippenko (1982), we show the parallactic angle as a function of declination for the latitude of the Keck Observatory $\left(19^{\circ} 49.6^{\prime}\right)$ in the top panel of Figure 4, while the rate of change of the parallactic angle is shown in the bottom panel. Declinations close to the latitude of the Observatory require relatively large rotation rates in order to compensate field rotation for small hour angles near the zenith. It is evident that nearly the entire sky is observable with field rotation rates less than $2 \%$ minute.

We investigated an intentionally extreme case of instrument-induced polarization variation during a model N\&S observation in Figure 5. We assume a K-mirror rotation rate of $0.5 \%$ minute, a polarized sky and a polarized K-mirror. Together these elements result in a sky background that varies by $100 \%$ over a $90^{\circ} \mathrm{K}$-mirror rotation. Figure 5 shows a N\&S exposure sequence, beginning and ending with 1-minute exposures of the sky background and otherwise containing a sequence of 2-minute exposures alternating between the science field and the background field. An overhead of 15 seconds is assumed to move the telescope and settle at each field location. The resulting sky subtraction systematic in this model case is $2 \times 10^{-4}$, and is an example of how N\&S can eliminate smooth variations by balancing the sky exposure in the science and background fields.

A second case of time variation results from K-mirror rotation of defocussed point images on the image slicer during long integrations. This scenario is depicted in Figure 6. As the hexagonal pupil rotates (at the same rate as the parallactic angle), vertices of the footprint can walk on and off of a slit edge. The effect is most extreme at the outer edges of the high resolution KCWI slicer, where the defocussed images are comparable in width to the slits. There should be no effect in the center of the slicer, where the images are in focus, or in areas of the slicer that completely contain the rotating pupil footprint. We have modeled the most extreme case of a hexagonal footprint (which will occur near the defocussed corners of the image slicer) with its inscribed circle tangent to the edge of the slit. Rotation of the pupil will cause a peak-to-valley modulation of the intensity of about $1.2 \%$ in this case. A 2 -minute cadence N\&S sequence would reduce the point source residuals in this severe case to below $1 \times 10^{-4}$, as long as the target is at least $10^{\circ}$ from the zenith. In the case of a spatially uniform sky, areas of the pupil that rotate off of a given slice are largely 
compensated by areas that rotate on from neighboring slices, reducing this effect close to zero, proportionate to the sliceto-slice efficiency variation.

\section{INSTRUMENT DESIGN}

KCWI is designed for operation on the Keck II telescope at the right Nasmyth focal station. Figure 7 shows a view of the instrument with the outer enclosure transparent. A mannequin is shown as an indication of the overall size. The instrument operates in a fixed gravity vector with the field rotation compensated by a K-mirror for the science FOV, and a rotating tracking assembly for the instrument's guider. A large custom made optical bench is used to mount all of the instrument's components, and three kinematic mounting points are used to accurately position the instrument at the telescope focus. Transport rails are provided on the Nasmyth platform and deck to allow the instrument to be moved between the focal station and a storage location, and the instrument includes a cart to allow the instrument to be moved using the transport rails.

The instrument is provided with a hermetic, positively pressurized enclosure to protect the optical components against dust and other contaminants. The blue channel of the spectrograph is mounted on the bottom of the optical bench, and all of the other components are mounted on the top. The electronics are mounted in an electrically shielded, glycol cooled equipment rack that provides service access to all of the electronics except for the CCD and guide camera controllers that are mounted as part of their related opto-mechanical assemblies.

The optical and mechanical configuration of KCWI is illustrated in block diagram form in Figure 8. The KCWI optical path starts with light arriving at the Keck II right Nasmyth focal station from the telescope's tertiary mirror. The center of the field passes through a window and then through a K-mirror image de-rotator that delivers a stationary, on-axis, unvignetted 40" diameter science field to the selectable IFU slicer stack located at the telescope focus. The window allows the entire instrument to be sealed and positively pressurized with clean dry air. The image slicers sit on a linear stage that selects among the 3 slicer formats or a direct imaging alignment camera. A calibration system located ahead of the telescope focus has a deployable periscope mechanism to direct calibration light onto the image slicer. A second actuator mechanism moves a set of calibration targets in front of the calibration source.

A tracking guider (inset, Figure 7) is located ahead of the K-mirror and samples a 3' x 3' field located 3.24' off axis. The tracking guider follows the guide stars as they rotate about the optical axis during observations. It utilizes a standard 3' MAGIQ guide camera (Adkins, 2008) that provides facilities for automatic focusing of the telescope and monitoring of image quality.

Light from the IFU slicer mirrors is reconfigured into a virtual long slit by the pupil mirror array. Our novel design will be fabricated by Winlight (France) and incorporates 3 selectable image slicers and a single pupil mirror array. The three selectable slicer mirror stacks provide $0.35 "$, 0.7 " or 1.4" spatial resolution (with corresponding 8.4", 16.8", or 33.6" FOV in one axis and a 20" FOV for all configurations in the other axis). The light from the pupil array proceeds to the spherical collimator, and then to a series of three fold mirrors. The spectrograph includes a selectable bandpass filter, a selectable, variable tilt grating, and an articulated CCD camera. The third fold mirror and the remainder of the blue channel optical path are located below the KCWI optical bench. The left panel of Figure 9 shows an isometric view of the KCWI optical layout. The fold mirror designated FMD will be replaced with a dichroic beam splitter when the red channel is added to the spectrograph. The key KCWI optical design parameters are summarized in Table 3.

KCWI bears strong resemblance to CWI. Both have a $150 \mathrm{~mm}$ spectrograph beam size and a demagnification of $\sim 8$. The main departures of KCWI result from the long path length needed to accommodate the 2-channel design and the superior imaging required by the highest spectral resolution mode matched to the excellent seeing at WMKO. These enhancements have been achieved by following a Schmidt camera design principle in the collimator, coupled with a wavefront-correcting cylindrical fold mirror.

While CWI employs a flat IFU and spherical collimator to simultaneously re-image the telescope pupil (close to infinity) onto a grating at its focal point while collimating the light from a straight virtual slit, KCWI introduces a very modestly convex ( $\sim 5 \mathrm{~m}$ radius) image slicer with a flat pupil mirror array to re-image the telescope pupil at the center of curvature of the spherical collimator. This provides the increased path length needed to accommodate the two KCWI channels separated vertically on the two sides of the instrument's optical bench. It follows from this that the KCWI virtual slit is curved, with a radius equal to the focal length of the collimating mirror (a Schmidt camera in reverse). This approach is 
capable of exquisite imaging performance, except that the collimator must be used $4.5^{\circ}$ off axis in order to steer the afocal beams clear of the IFU. This introduces some aberrations into the wavefront, and these are compensated by applying a slight cylindrical figure to the FM1 fold mirror. The required curvature amounts to only $\sim 16 \mu \mathrm{m}$ depth or a radius of curvature of about a 0.7 kilometer on the short axis of the mirror. Mirrors of this design are unusual in the optical band, but common in x-ray optics. Winlight, (the IFU vendor) has manufactured similar cylindrical mirrors with tighter wavefront quality requirements than $\mathrm{KCWI}(\sim 0.5$ waves per $150 \mathrm{~mm})$.

Table 3: Key KCWI Optical Parameters

\begin{tabular}{ll}
\hline Parameter & Value \\
\hline Bandpass (blue channel) & 350 to $560 \mathrm{~nm}$ (acceptable performance to $700 \mathrm{~nm})$ \\
\hline Field of View & $20 " \mathrm{x}(8.3,16.5$, or 33)" selectable \\
\hline Telescope plate scale & $1.375 \mathrm{/pixel}$ \\
\hline Detector plate scale & $0.15 \mathrm{"} / \mathrm{pixel}$ \\
\hline IFU pupil array radius & $225 \mathrm{~mm}$ \\
\hline IFU slicer radius & $5200 \mathrm{~mm}$ \\
\hline Aberration correction & Cylindrical fold mirror \\
\hline Slit height & $14.5 \mathrm{~mm}$ \\
\hline Slit width & $0.25,0.5,1 \mathrm{~mm}$ selectable \\
\hline Beam size & $150 \mathrm{~mm}$ \\
\hline Collimator focal length & $2160 \mathrm{~mm}$ \\
\hline Camera focal length & $305 \mathrm{~mm}$ \\
\hline Gratings & $\mathrm{VPH}, 250 \times 350 \mathrm{~mm}$, selectable \\
\hline Blue detector & $\mathrm{e} 2 \mathrm{v} 4 \mathrm{kx} 4 \mathrm{k}, 15 \mu \mathrm{m}$ pixel \\
\hline Image resolution & $\sim 10 \mu \mathrm{m} \mathrm{RMS} \mathrm{(seeing-limited)}$ \\
\hline
\end{tabular}

The remaining large optics downstream of FM1 are flat mirrors. FMD will eventually be replaced by a dichroic to accommodate the red channel. For the time being, it will be a low risk flat coated with broad-band enhanced silver to maximize the wavelength coverage of the blue channel. Optimized Ag coatings throughout the instrument will enable good performance out to $700 \mathrm{~nm}$, beyond which the performance of the blue camera and the blue-optimized CCD will begin to fall.

On the lower side of the bench, the flat fold mirror FM3 redirects the beam through a blocking filter to the grating. The gratings in each channel are located near the collimator center of curvature with the result that the beams converge to form a common pupil that is close in diameter to the $150 \mathrm{~mm}$ spectrograph beam size. Gratings and filter elements are exchanged using a single jukebox mechanism shown in Figure 10. Each element is held in a storage rack. The slots in the storage rack are provided with support rollers to hold each element at the top and separator plates that prevent contact between adjacent elements. The storage rack is translated back and forth by a screw-driven linear mechanism to position the selected element for withdrawal from the storage rack. Once the element is withdrawn another screw mechanism raises the element to define it against a kinematic mount located above at the required in beam position. The kinematic mount for the grating also provides the rotation drive to adjust the tilt of the grating. The jukebox mechanisms are designed so that positive engagement with the filter or grating is maintained during all phases of motion, protecting 
the optics from damage due to power or other failures, or earthquakes. The detailed design phase of KCWI will include demonstration of this key element, which is currently in the final stages of drafting.

Our preliminary suite of five blue Volume Phase Holographic (VPH) gratings is shown in Table 4. A red-optimized grating may also be procured depending on the expected construction phasing of the red channel. The gratings are each designed to meet the instrument resolution requirements, maximize efficiency, and minimize stray and scattered light, while also conforming to the mechanical constraints imposed by the opto-mechanical implementation. A matched set of blocking filters is also accommodated by the instrument design. The advantages of VPH gratings for astronomical applications over the classical surface relief (SR) designs have been discussed in literature (for example Barden, 2000). The key characteristics of VPH gratings are high diffraction efficiency, high resolving power, low stray and scattered light, and tunable bandpass. Separate gratings are designed for the high, moderate, and low resolution applications. All of the gratings are designed to operate in an efficiency range of 65 to $90 \%$.

Table 4: Preliminary KCWI Blue Grating Set

\begin{tabular}{r|cccccccc|}
\multicolumn{1}{r}{ Grating } & \multicolumn{2}{|c}{ Bandpass (nm) } & \multicolumn{3}{c}{ Wavelength $(\mathbf{n m})$} & \multicolumn{3}{c|}{ Resolution $(\boldsymbol{\lambda} / \boldsymbol{\Delta} \boldsymbol{\lambda})$} \\
\hline & N\&S & CCD & Min. & Peak & Max. & Min. & Peak & Max. \\
BH1 & 13 & 41 & 350 & 381 & 415 & 4300 & 6200 & 5300 \\
BH2 & 15 & 44 & 410 & 446 & 485 & 4300 & 6200 & 5300 \\
BH3 & 18 & 53 & 470 & 515 & 560 & 4300 & 6200 & 5300 \\
BM1 & 30 & 90 & 350 & 410 & 560 & 1700 & 3000 & 2300 \\
BL1 & 70 & 200 & 350 & 425 & 560 & 750 & 1200 & 970 \\
\hline
\end{tabular}

In order to maximize efficiency, the tilt of each grating may be adjusted to access the desired wavelength range. The camera is also articulated on curved rails around the grating axis of rotation using the camera articulation stage shown in Figure 10. The KCWI design permits articulation from the direct imaging (zero order) position through an arc of $120^{\circ}$. The camera articulation stage is driven by a rotary actuator and gear pinion that engages a rack centered between the curved rails. The rack and pinion is selected to have the low backlash needed to provide the positioning resolution and stability required by the spectrograph (a fraction of a pixel). A magnetic absolute position linear encoder will be used as the feedback for the position control loop, which allows the camera position to be determined without homing.

Dispersed light from the grating is reimaged with a custom, all-spherical camera lens optimized for the band of interest and coupled to a liquid nitrogen cooled CCD detector. The last lens of the camera is used as the vacuum window. The $\mathrm{CCD}$ detector is designed for operation between $-100{ }^{\circ} \mathrm{C}$ and $-140{ }^{\circ} \mathrm{C}$, with a minimum hold time of 30 hours. A focus mechanism allows precise positioning of the detector and window behind the camera barrel. The deployable nod and shuffle mask (Figure 2) is driven through a ferrofluidic vacuum feedthrough. We have implemented a demonstration version of this mask, shown in Figure 10, in the CWI instrument at Palomar Observatory. The KCWI blue detector is a production e2v CCD231-84, the same as CWI. These quiet devices have read noise in the 2 to 3 electron range and quantum efficiency (QE) from 60 to $85 \%$ over the wavelength range of 350 to $700 \mathrm{~nm}$. The typical QE of the CCD23184 is shown in Figure 11.

The camera design (shown in the right panel of Figure 9) includes a minimum number of elements to provide high UV transmission. Calcium Fluoride is the only practical choice to generate most of the positive optical power, by reason of its low dispersion. Ohara i-line glasses are used for color correction. They are specifically formulated and manufactured so as to yield maximum internal UV transmission. Ohara BSM51Y is used for the field flattener/vacuum window as it is known to be free of Potassium 40 and other radioactive substances. Nine free-standing, all-spherical lens elements were required. The quartet, triplet, doublet configuration shown produced the best imaging over the full 350 to $560 \mathrm{~nm}$ design wavelength range without refocus, with acceptable performance to $700 \mathrm{~nm}$ (providing extended response prior to the phased inclusion of the dichroic beamsplitter and red channel). The performance of this $223 \mathrm{~mm}$ diameter, $305 \mathrm{~mm}$ focal length camera results an average rms image diameter without refocus of $15.0 \pm 4.4 \mu \mathrm{m}$, maximum rms lateral color for direct imaging of $7.1 \mu \mathrm{m}$, distortion at full diagonal field of $0.36 \%$. The polychromatic imaging pupil is $158.0 \mathrm{~mm}$ in diameter and the spectral imaging is pixel-sampling limited.

The model end-to-end optical performance of the KCWI blue channel design is illustrated in Figure 12. KCWI can attain an absolute spatial resolution $(<10 \mu \mathrm{m}$ rms radius), 2 to 3 times better than CWI, enabling it to perform high resolution spectroscopy $(\mathrm{R} \sim 20,000)$ and sky-limited direct imaging. It can also attain a compound pupil $<160 \mathrm{~mm}$ 
diameter at the grating in both channels, independently of the selected image slicer. This is achieved with a pupil reimaging IFU, Schmidt camera approach, and aberration-correcting cylindrical fold mirror FM1. With this performance, the transfer function of the KCWI optics will negligibly affect the image width of the narrowest slit (35 $\mu \mathrm{m}$ at the CCD) or the stellar image presented by the telescope ( $\sim 39 \mu \mathrm{m}$ radius at the CCD if the seeing is $0.4 ")$. This performance can be achieved across the long virtual slit in both bands.

KCWI will maximize its throughput through the use of custom coatings, including Sol-gel and enhanced Al/Ag coatings available from the labs at University of California, Santa Cruz (UCSC) and also from commercial vendors. The use of custom coatings allows the instrument's 9 broad-band reflections to be optimized for incidence angle (for example in the K-mirror assembly) and balanced to maintain high blue efficiency while supporting the future addition of the red arm without any re-coating. KCWI's long-term efficiency will be preserved by the use of a hermetic enclosure to protect the optics from contamination.

\section{SUMMARY}

KCWI is a powerful new integral field spectrograph for the W. M. Keck Observatory. We are currently completing the detailed design phase, and are funded by the NSF through the TSIP program for construction of the blue channel of the instrument. We are actively seeking funding for construction of the red channel.

$\mathrm{KCWI}$ is designed to detect faint diffuse emission at a level less than $1 \%$ of the sky background, and it achieves this with a low flexure, bench mounted optical design, minimal vignetting, and a nod and shuffle observing mode designed to reduce systematics. The instrument is designed to meet the observing requirements of a large range of observing programs by providing configurable spectral resolution, spectral range, and seeing-limited imaging.

This material is based upon work supported by AURA through the National Science Foundation under Scientific Program Order No. 5 as issued for support of the Telescope Systems Instrumentation Program (TSIP), in accordance with Proposal No. AST-0335461 submitted by AURA. We also acknowledge and thank the Caltech Optical Observatories for continued support of both CWI and KCWI.

\section{REFERENCES}

[1] Martin, D. C., “The Keck Cosmic Web Imager,” Proc. SPIE 7735, 77350M, 2010.

[2] Matuszewski, M., "The Cosmic Web Imager: an integral field spectrograph for the Hale Telescope at Palomar Observatory: instrument design and first results," Proc. SPIE 7735, 77350P, 2010.

[3] Bertone, S., and Schaye, J., "Rest-frame UV line emission from the intergalactic medium at $2 \leq z \leq 5$," Mon. Not. R. Astron. Soc., 419: 780-798, 2012.

[4] Glazebrook, K., and Bland-Hawthorn, J., "Microslit Nod-Shuffle Spectroscopy: A technique for Achieving Very High Densities of Spectra," PASP, 113:197-214, 2001.

[5] Patat, F., "The dancing sky: 6 years of night sky observations at Cerro Paranal," A\&A, 481:575-591, 2008.

[6] Filippenko, A., "The Importance of Atmospheric Differential Refraction in Spectrophotometry," PASP, 94:715-721, 1982.

[7] Adkins, S., "MAGIQ at the W. M. Keck Observatory: Initial deployment of a new acquisition, guiding, and image quality monitoring system," Proc. SPIE 7014, 70141U, 2008.

[8] Barden, S., "Volume Phase Holographic Gratings and the Efficiency of Three Simple Volume Phase Holographic Gratings," PASP, 112:809-820, 2000. 

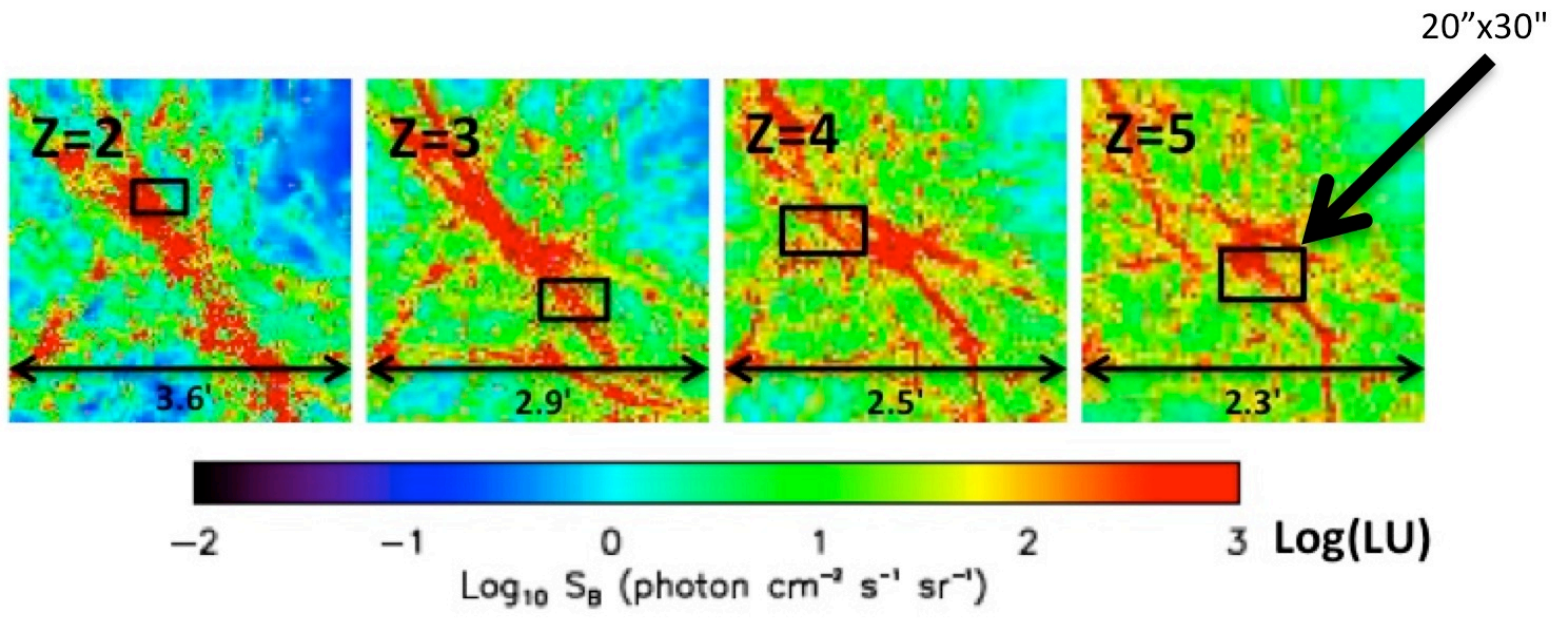

Figure 1: Simulations of cosmic web hydrogen Lyman alpha emission as a function of redshift reproduced from Bertone and Schaye (2012). The black box is representative of the projected size of the large KCWI image slicer on the sky.

$4 \mathrm{k} \times 4 \mathrm{k} C \mathrm{CD}$

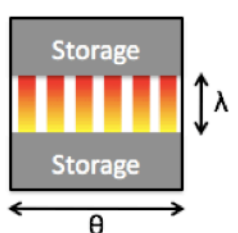

$\theta$

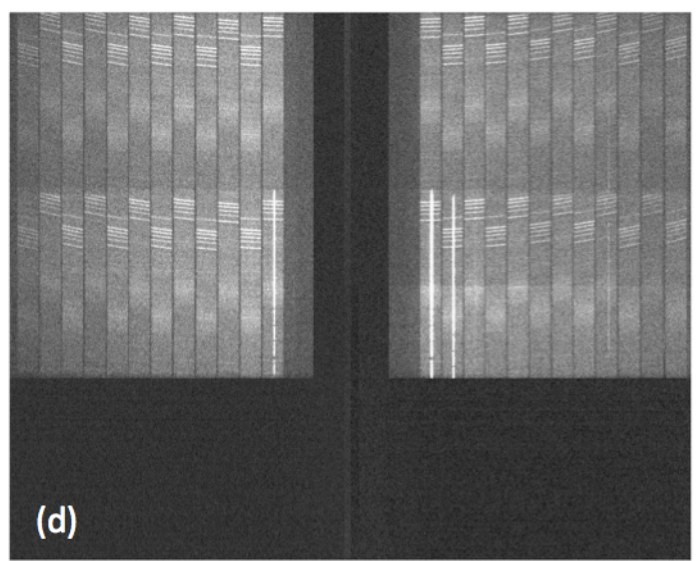

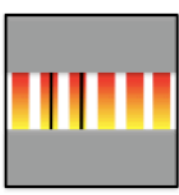

(a)

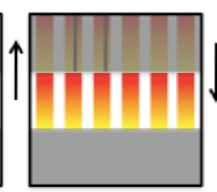

(b)

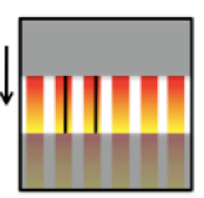

(c) (e)

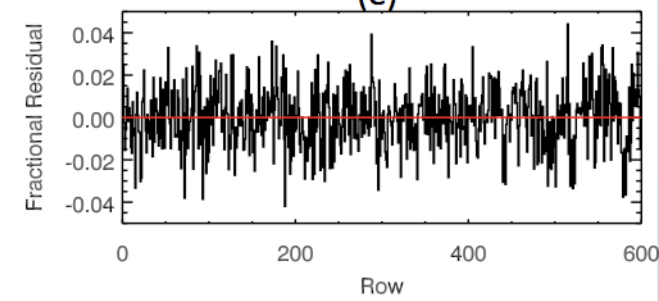

(f)

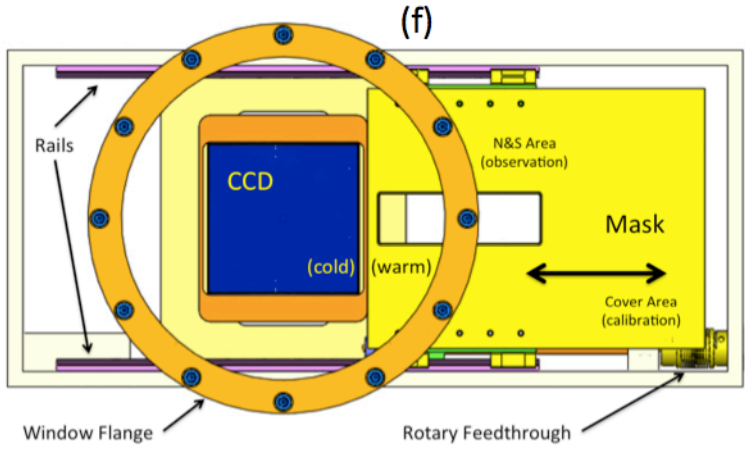

Figure 2: Nod and shuffle (N\&S) operation. (a) source region observed (e.g., 2 min.); (b) shutter closed, tel. nodded to background region, CCD source charge shuffled behind N\&S mask, shutter opened, background observed for equal time (e.g., 2 min.); (c) shutter closed, tel. back to source region, CCD shuffled to return source charge to open area, another exposure; and so on for $\sim 10$ to 20 cycles. (d) Source image in central 1/3, sky in upper 1/3. (e) Subtraction residuals in test N\&S observation without nodding. Residuals are consistent with photon plus read noise. (f) Layout of N\&S mask mechanism. 


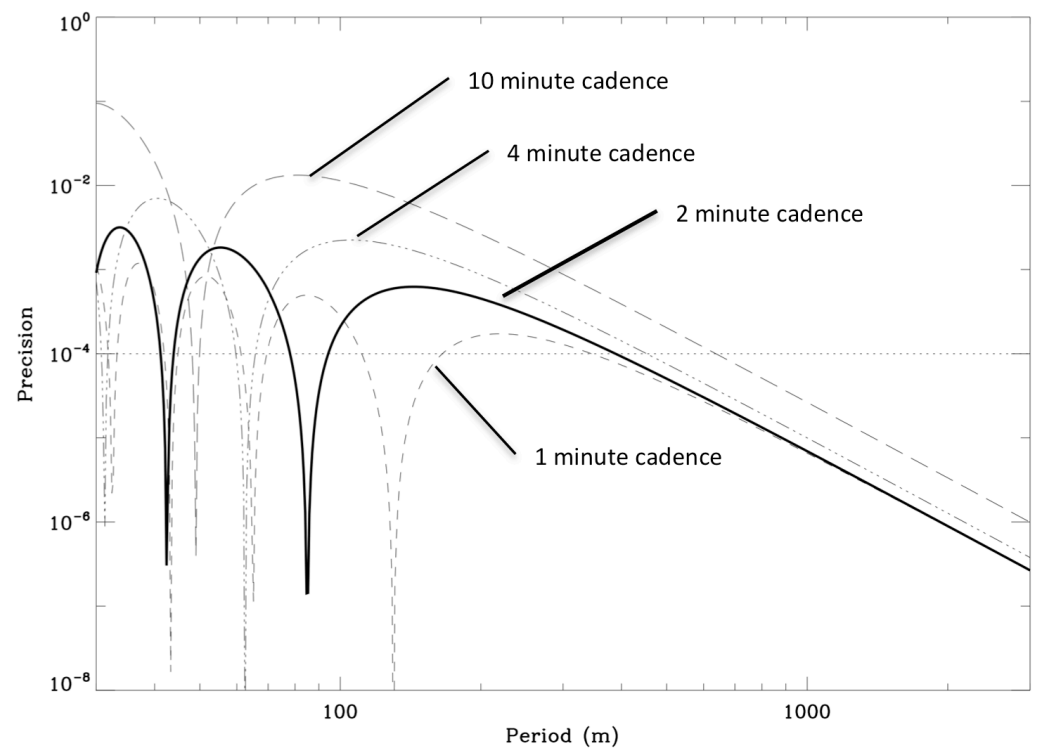

Figure 3: Modeled sky subtraction precision as a function of sky variation time period given several nod and shuffle cadences for a 40 minute total (sky+background) integration. The modeled sky variations are sinusoidal with an amplitude of $+/-10 \%$.

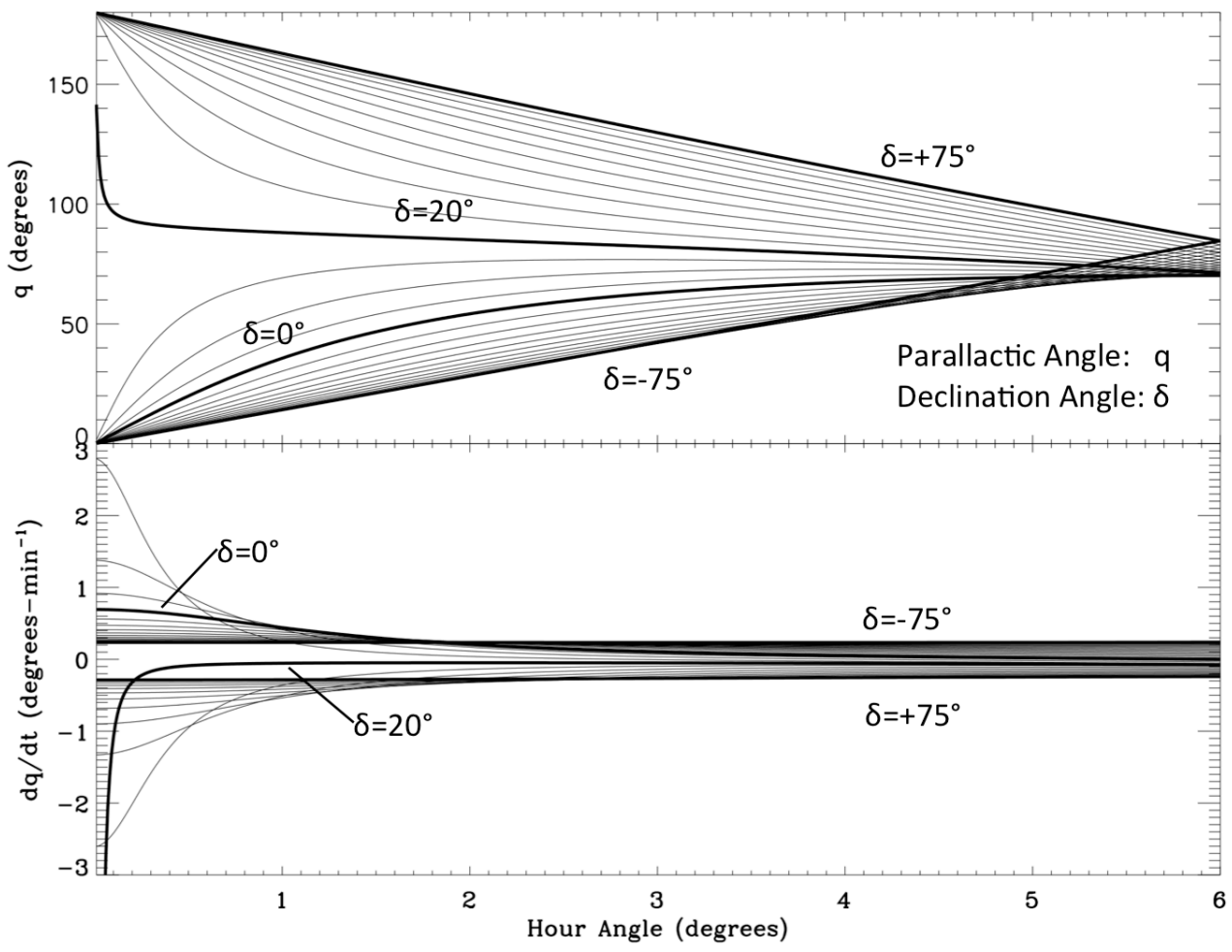

Figure 4: (Top panel) Parallactic angle (q) vs hour angle for a range of declinations at the latitude of the Keck Observatory $\left(\sim 20^{\circ}\right)$. (Bottom panel) Corresponding field rotation rates for a range of declinations $(\delta)$ and hour angles viewed from Keck Observatory. The K-mirror rate is half of the rate of the parallactic angle, dq/dt. 


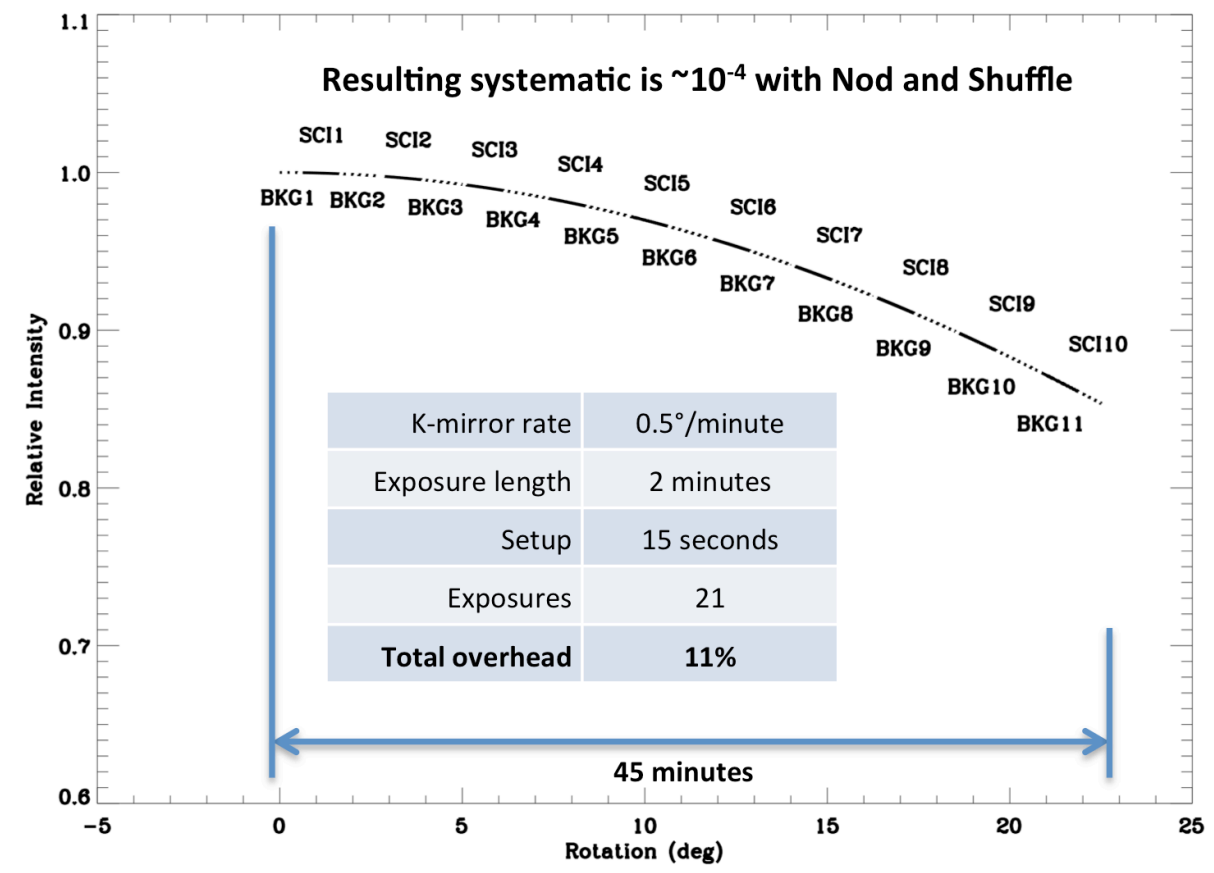

Figure 5: A model nod and shuffle observation with a polarized sky background and K-mirror derotator. 21 images are taken in sequence. The first and last exposures are of the background field and are 1 minute in length. The remaining exposures are each 2 minutes long, alternating between the science field and the background field. A delay of 15 seconds is assumed between each exposure. The CCD is read out only once at the end of the series. The integrated sky and background exposures will subtract with a precision of close to $10^{-4}$ in this scenario at the cost of $11 \%$ overhead, compared to a precision of only $\sim 10^{-2}$ for individual exposure pairs.

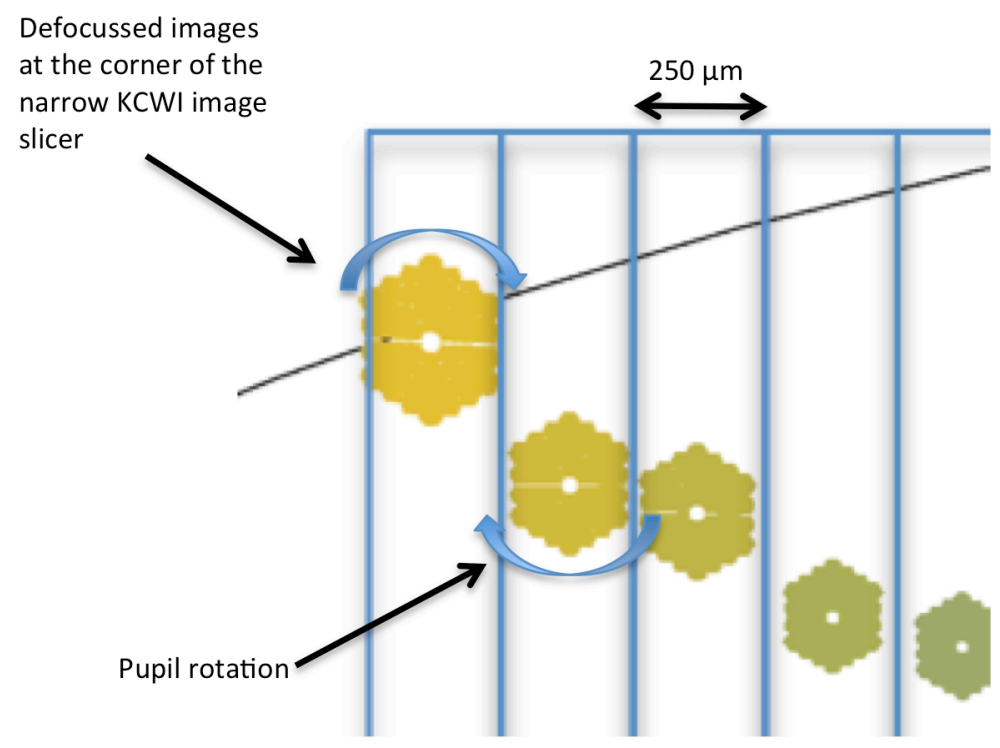

Figure 6: Pupil rotation during KCWI images can affect point source photometry near defocussed slit edges (particularly with the high resolution slicer), but is largely cancelled for diffuse fields. The effect is reduced near the slicer center. 


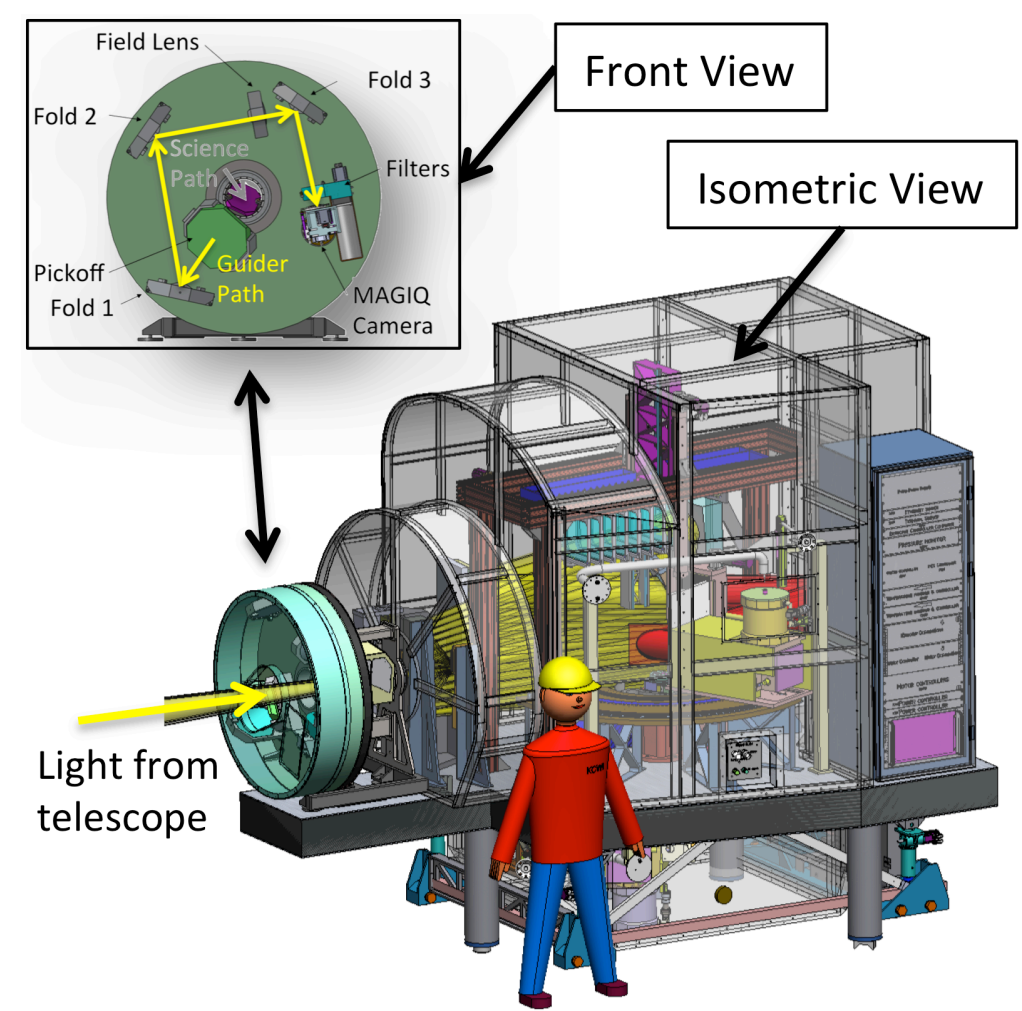

Figure 7: A view of the KCWI instrument with transparent enclosure. Large optics common to both channels, electronics, and the red spectrograph are on the top of the bench, while the blue channel is underneath. The inset shows the tracking guider, which follows guide stars around the optical axis of the telescope.

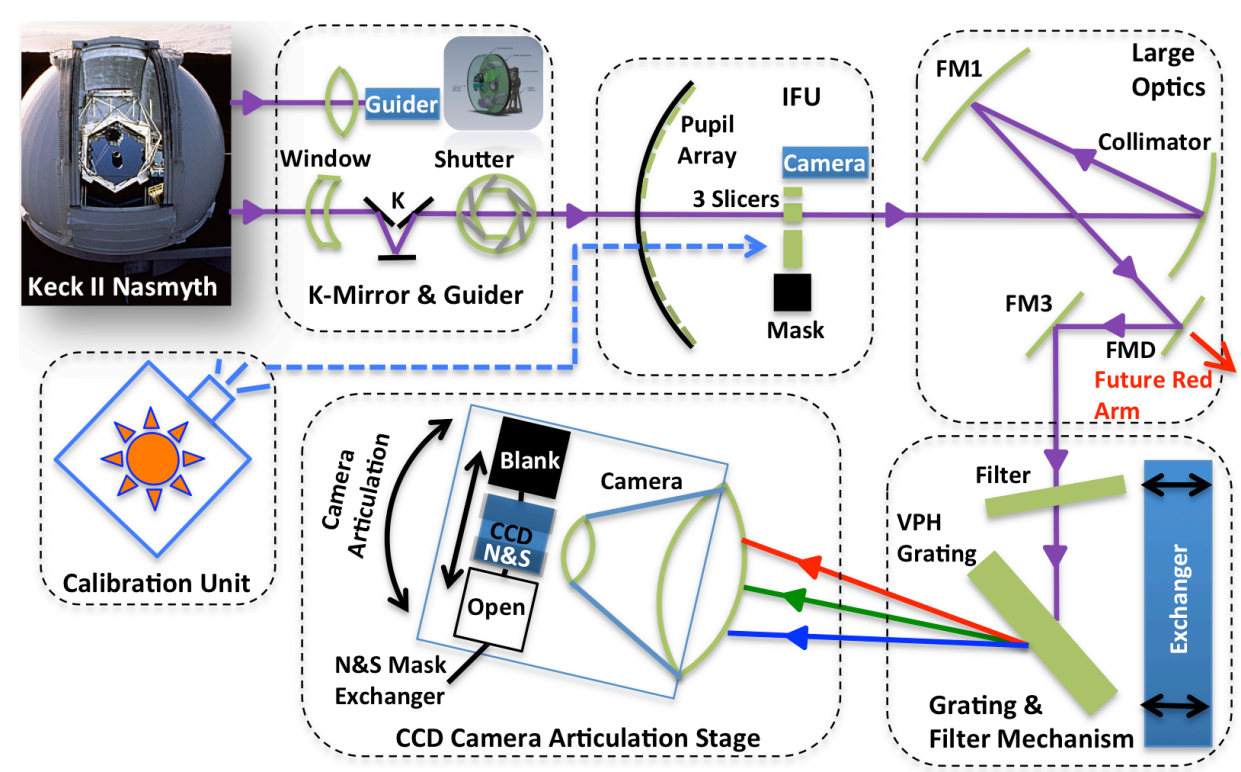

Figure 8: A block diagram view of the KCWI instrument. The blue implementation will employ a simple flat mirror FMD, which will be replaced by a dichroic beamsplitter during the installation of the red channel. 


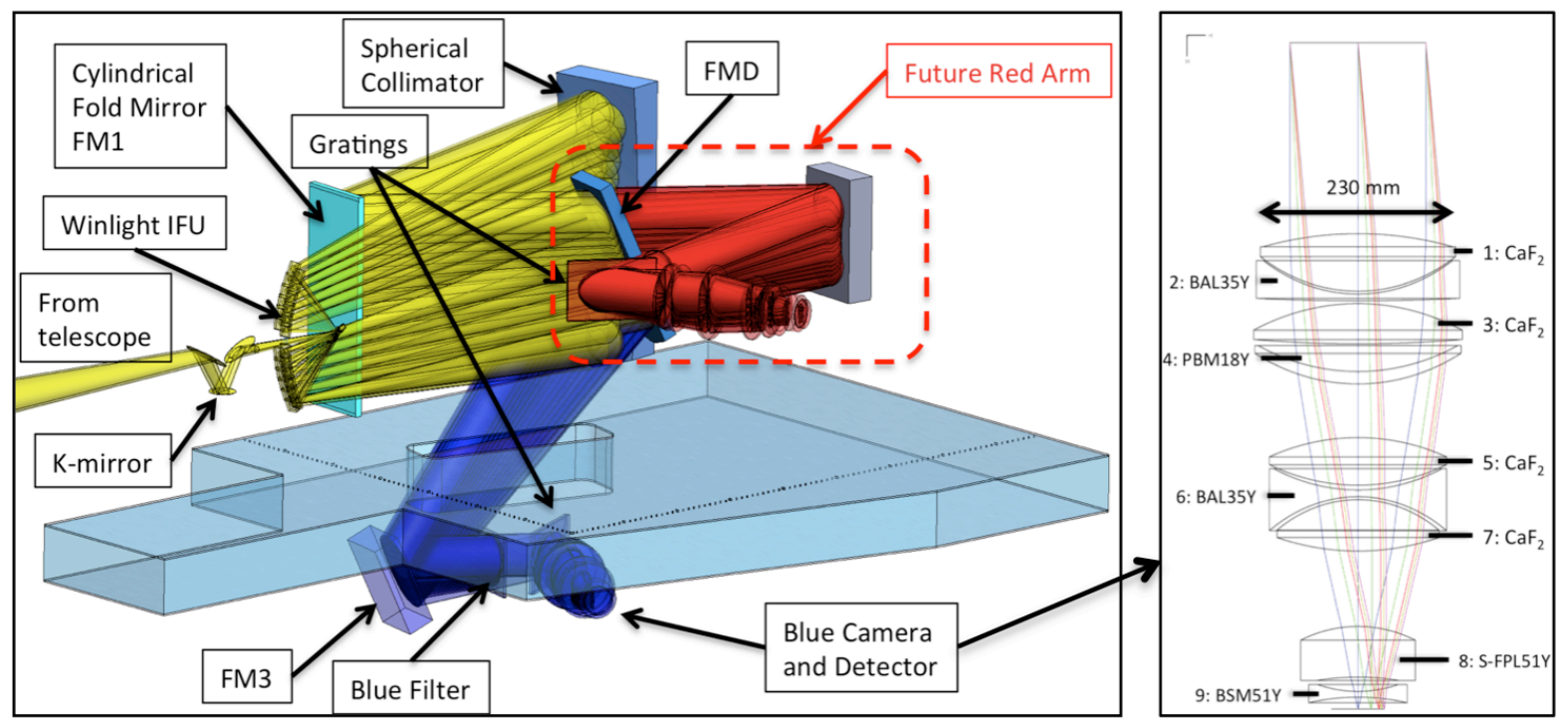

Figure 9: (Left Panel) The KCWI optical layout showing the portion of the optical path common to both channels in yellow. The red and blue arms of the spectrograph are separated by a dichoic beamsplitter. (Right panel) The preconstruction blue channel camera design.

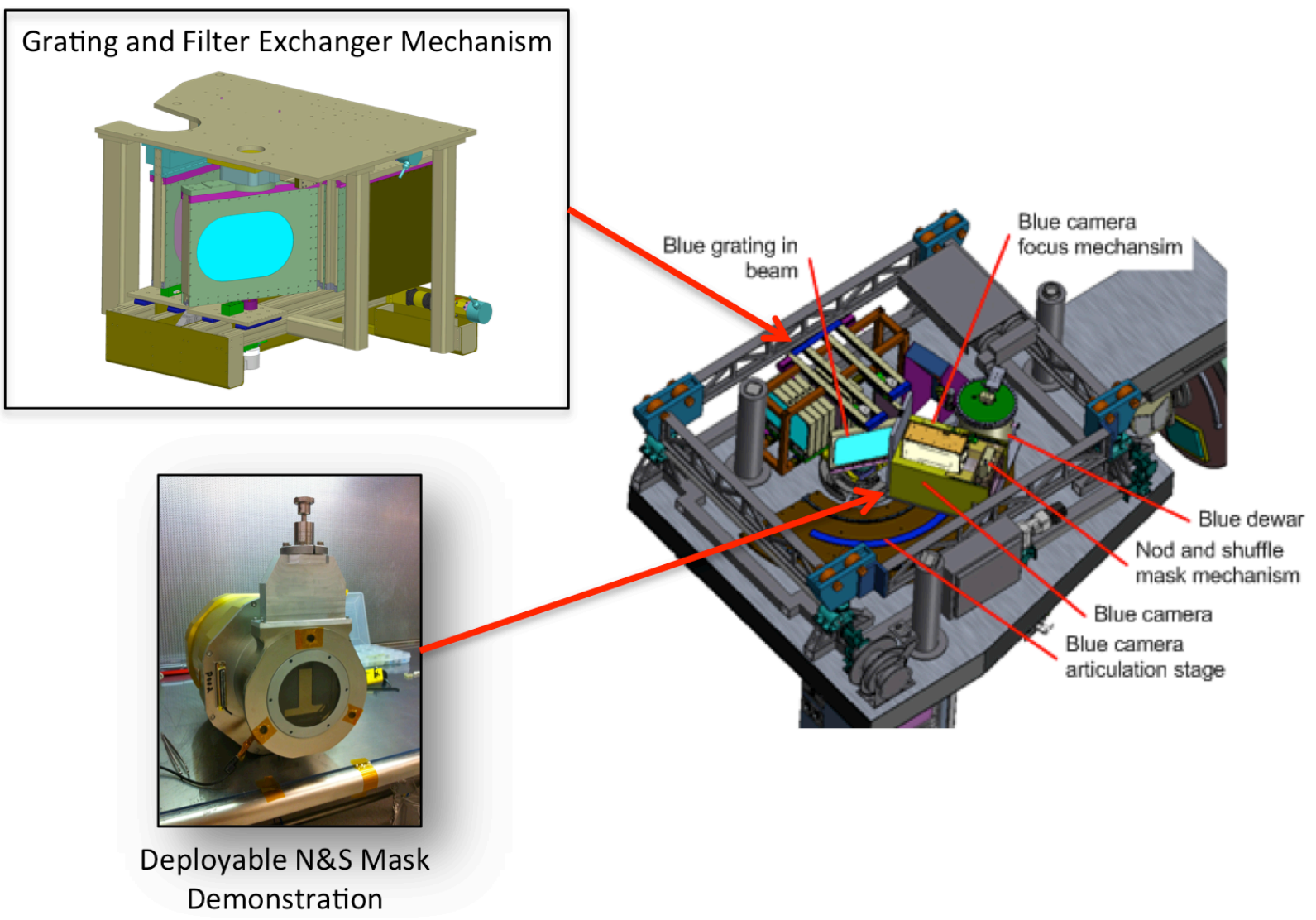

Figure 10: The KCWI detailed design includes a demonstration of two mechanisms. The deployable N\&S mask has already been implemented on CWI at Palomar Observatory, while the blue grating exchanger mechanism is in the final stages of drafting for fabrication. 


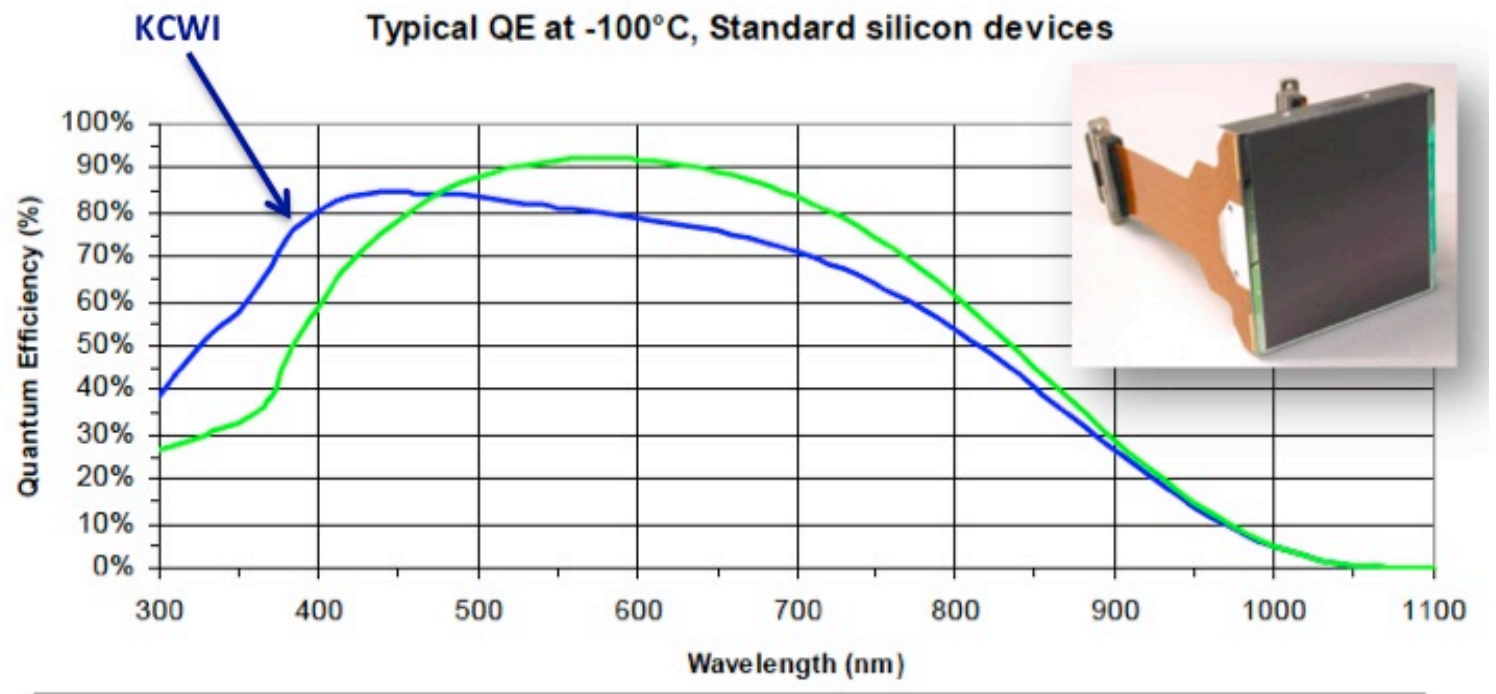

Figure 11: The KCWI blue channel uses a production e2v CCD231-84. These devices are anti-reflection coated for excellent blue quantum efficiency. The device is usable through the visible band.

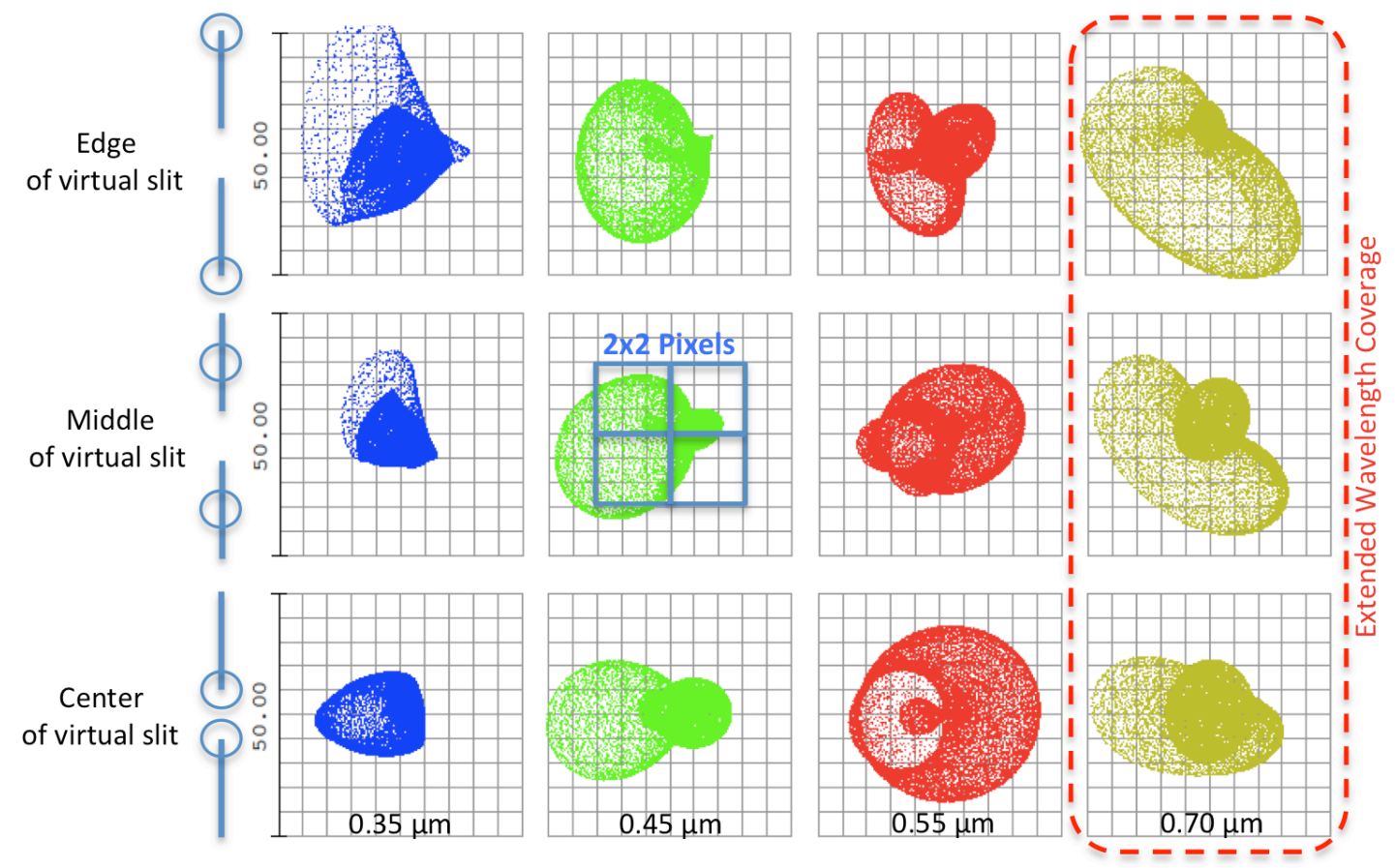

Figure 12: Modeled KCWI end-to-end optical performance showing image quality along the virtual slit for a range of wavelengths, including extended performance to $700 \mathrm{~nm}$ while awaiting installation of the red channel. 